\title{
Maximum tumor diameter is an independent predictor of prostate-specific antigen recurrence in prostate cancer
}

\author{
Lori E Eichelberger ${ }^{1}$, Michael O Koch², John N Eble ${ }^{1}$, Thomas M Ulbright ${ }^{1}$, Beth E Juliar ${ }^{3}$ \\ and Liang Cheng ${ }^{1,2}$ \\ ${ }^{1}$ Department of Pathology and Laboratory Medicine, Indiana University School of Medicine, Indianapolis, IN, \\ USA; ${ }^{2}$ Department of Urology, Indiana University School of Medicine, Indianapolis, IN, USA and ${ }^{3}$ Division of \\ Biostatistics, Indiana University School of Medicine, Indianapolis, IN, USA
}

\begin{abstract}
Maximum tumor diameter has been shown to correlate with multiple predictors of clinical outcome in prostate cancer. In the current study, we prospectively analyze whether maximum tumor diameter is a significant predictor of prostate-specific antigen (PSA) recurrence. The study population consisted of 364 patients who underwent radical prostatectomy for prostate cancer. Prostatectomy specimens were evaluated by wholemount processing of the entire prostate. Maximum tumor diameter was measured from the whole-mount sections of the prostate. Spearman's coefficient of rank correlation was used to correlate tumor diameter with continuous variables. $T$-tests or analysis of variance (ANOVA) tests were performed to determine if tumor diameter was significantly associated with other clinical and pathologic variables. The effect of clinical and pathologic variables on time to recurrence was analyzed using Cox regression. The mean tumor diameter for all patients was $1.73 \mathrm{~cm}$ (range, $0.02-4.40 \mathrm{~cm}$ ). Maximum tumor diameter was associated with preoperative PSA $(r=0.22, P<0.0001)$, prostate weight $(r=-0.12, P=0.028)$, tumor volume $(r=0.87, P<0.0001)$, Gleason score $(r=0.29, P<0.0001)$, and pathologic stage $(P<0.0001)$. Cox multiple regression was performed to test the prognostic value of maximum tumor diameter adjusting for pathologic stage, Gleason score, and surgical margin status. Increased maximum tumor diameter was associated with shorter time to PSA recurrence (hazard ratio $=1.70,95 \%$ confidence interval 1.13-2.56, $P=0.01$ ), controlling for risk factors, Gleason score, and surgical margin status. We conclude that maximum tumor diameter is a significant predictor of biochemical recurrence in patients with prostate cancer.
\end{abstract}

Modern Pathology (2005) 18, 886-890. doi:10.1038/modpathol.3800405; Published online 1 April 2005

Keywords: prostate; neoplasm; prostatectomy; tumor length; tumor diameter; tumor volume; PSA recurrence; TNM staging

Numerous studies of prostate carcinoma have investigated measurement of tumor size and its impact on prognosis. Multiple histologic techniques have been utilized to assess tumor size since the gross features of prostatic carcinoma are subtle ${ }^{1}$ and the tumors are often multifocal. ${ }^{2-4}$ Maximum tumor diameter has been suggested as a fast, easy, and objective method of estimating the amount of tumor. Renshaw et $a l^{5}$ demonstrated that measurements of the maximum diameter of the largest focus of tumor, the largest single tumor area, and the sum of the

Correspondence: Dr L Cheng, MD, Department of Pathology and Laboratory Medicine, Indiana University Medical Center, University Hospital 3465, 550 North University Blvd., Indianapolis, IN 46202, USA.

E-mail: lcheng@iupui.edu

Received 9 September 2004; revised and accepted 20 January 2005; published online 1 April 2005 areas of two separate tumor foci correlated with tumor volume. Maximum tumor diameter has also been shown to correlate with other predictors of clinical outcome, including surgical margin status, preoperative prostate-specific antigen (PSA) levels, Gleason score, and pathologic stage. ${ }^{6}$ In this study, we analyzed radical prostatectomy specimens by whole-mount processing to determine if maximum tumor diameter is a significant predictor of PSA recurrence.

\section{Methods}

In all, 364 patients were included in the study and treated by radical retropubic prostatectomy from 1999 to 2003 at Indiana University Hospital. Patients who received preoperative radiation or 
androgen deprivation therapy were excluded. Serum PSA was measured using the Immulite $^{\mathbb{B}}$ PSA assay (Diagnostics Products Corporation, Los Angeles, CA, USA). The study population was prospectively followed for PSA recurrence, defined as a serum PSA value of at least $0.1 \mathrm{ng} / \mathrm{ml}$ after surgery. ${ }^{7-9}$ PSA levels were obtained at $1,3,6,9,12$, 18 , and 24 months following radical prostatectomy. Thereafter, follow-up was adjusted to clinical situation, but was at least annual. The median length of follow-up was 12 months (range, 1.5-48 months; mean, 14 months). This research was approved by the Indiana University Institutional Review Board.

The radical prostatectomy specimens were examined as previously described. ${ }^{6}$ All pathologic data, including tumor diameter, were collected prospectively by a single pathologist (LC). Prostates were weighed, measured, inked, and fixed in 10\% neutral formalin. Following fixation, the apex and base were amputated and serially sectioned at $3-5 \mathrm{~mm}$ intervals in the vertical, parasagittal plane. The seminal vesicles were sectioned parallel to their junction with the prostate and entirely submitted for examination. The remaining prostate was serially sectioned perpendicular to the long axis from the apex of the prostate to the base, and whole-mount sections were prepared. The greatest diameter of the largest single focus of tumor was obtained by marking both ends of the tumor on the glass slide utilizing a $\times 4$ objective, and measuring this distance with a ruler marked with millimeters. If the tumor size was $<0.5 \mathrm{~cm}$, an ocular micrometer was used. If tumor was present in consecutive sections in the same orientation and location, the tumor was viewed as contiguous from section to section. The thicknesses of the sections involved were summed and constituted the greatest diameter, if larger than the visualized tumor on two-dimen- sional slides. The volume of carcinoma in the entire prostate was determined by the grid method and was the sum of the volumes of individual foci of tumor. ${ }^{6}$ In this method, the sum of each area was multiplied by the thickness of the average slice, and the sum of these volumes was multiplied by a factor of 1.25 to account for tissue shrinkage during processing. ${ }^{10}$

The 1997 TNM (tumor, nodes, and metastasis) system was used for pathologic staging. ${ }^{11}$ We chose to use the 1997 TNM staging system since our recent study indicated that $2002 \mathrm{pT} 2 \mathrm{~b}$ tumors probably do not exist. ${ }^{10}$ Cancers were graded and scored according to the Gleason system. ${ }^{12,13}$ Surgical margins were considered positive when carcinoma cells were in contact with the inked margin. ${ }^{14}$

Statistical tests were conducted as two-sided at the 0.05 significance level and 0.10 marginal significance level. Spearman's coefficient of rank correlation was used to correlate diameter with continuous variables. $T$-tests or analysis of variance (ANOVA) tests were performed to determine if maximum tumor diameter was significantly associated with other clinical and pathologic variables. The effects of clinical and pathologic variables on time to PSA recurrence were analyzed using Cox multiple regression. Time to recurrence was evaluated using the Kaplan-Meier method.

\section{Results}

The association of maximum tumor diameter with patient and tumor characteristics are summarized in Tables 1 and 2. Preoperative PSA levels ranged from 0.3 to $150.0 \mathrm{ng} / \mathrm{ml}$ (median, $8.4 \mathrm{ng} / \mathrm{ml}$ ). Final pathologic $\mathrm{T}$ classifications were pT2a $(53,15 \%)$, pT2b $(220,60 \%)$, pT3a $(74,20 \%)$, and pT3b $(17,5 \%)$. The

Table 1 Correlation between patient characteristics and maximum tumor diameter

\begin{tabular}{|c|c|c|c|c|}
\hline \multirow[t]{2}{*}{ Characteristics } & \multicolumn{2}{|c|}{ Maximum tumor diameter $(\mathrm{cm})^{\mathrm{a}}$} & \multirow[t]{2}{*}{ Spearman's correlation coefficient } & \multirow[t]{2}{*}{ P-value } \\
\hline & $\begin{array}{c}\leq 1.7 \mathrm{~cm} \\
(n=192)\end{array}$ & $\begin{array}{l}>1.7 \mathrm{~cm} \\
(n=172)\end{array}$ & & \\
\hline \multicolumn{5}{|l|}{ Age (years) } \\
\hline Mean (range) & $60(41-77)$ & $60(43-76)$ & -0.01 & 0.796 \\
\hline \multicolumn{5}{|c|}{ Preoperative PSA $(\mathrm{ng} / \mathrm{ml})$} \\
\hline Mean (range) & $6.72(0.28-58.00)$ & $10.22(1.30-150.00)$ & 0.22 & $<0.0001$ \\
\hline \multicolumn{5}{|c|}{ Prostate weight (g) } \\
\hline Mean (range) & $45.17(15.30-149.00)$ & $39.34(14.00-103.00)$ & -0.12 & 0.002 \\
\hline \multicolumn{5}{|l|}{ Gleason score } \\
\hline Mean (range) & $6.29(5.00-9.00)$ & $6.73(4.00-9.00)$ & 0.29 & $<0.0001$ \\
\hline \multicolumn{5}{|c|}{ Tumor volume $\left(\mathrm{cm}^{3}\right)$} \\
\hline Mean (range) & $1.03(0.03-5.10)$ & $4.13(0.72-38.00)$ & 0.87 & $<0.0001$ \\
\hline
\end{tabular}

${ }^{\mathrm{a}}$ Tumor length was dichotomized by the mean maximum tumor diameter $(1.7 \mathrm{~cm})$ for illustrative purposes only; the data were analyzed as a continuous variable. The reported $P$-value is testing if correlation is significantly different than zero. 
Table 2 Association of patient characteristics with maximum tumor diameter

\begin{tabular}{|c|c|c|c|}
\hline \multirow[t]{2}{*}{ Characteristics } & \multicolumn{3}{|c|}{ Maximum tumor diameter $(\mathrm{cm})$} \\
\hline & $\mathrm{n}$ & Mean (SD) & P-value* \\
\hline Pathologic stage & & & $<0.0001$ \\
\hline pT2a and pT2b & 273 & $1.40(0.69)$ & \\
\hline рТЗа & 74 & $2.44(0.70)$ & \\
\hline pT3b & 17 & $2.81(0.60)$ & \\
\hline Multifocality & & & 0.220 \\
\hline Negative & 54 & $1.61(0.94)$ & \\
\hline Positive & 310 & $1.76(0.80)$ & \\
\hline Extraprostatic extension & & & $<0.0001$ \\
\hline Negative & 276 & $1.48(0.70)$ & \\
\hline Positive & 88 & $2.51(0.70)$ & \\
\hline Seminal vesicle invasion & & & $<0.0001$ \\
\hline Negative & 346 & $1.68(0.80)$ & \\
\hline Positive & 18 & $2.81(0.59)$ & \\
\hline Surgical margins & & & $<0.0001$ \\
\hline Negative & 278 & $1.57(0.76)$ & \\
\hline Positive & 86 & $2.27(0.79)$ & \\
\hline Perineural invasion & & & $<0.0001$ \\
\hline Negative & 77 & $0.88(0.55)$ & \\
\hline Positive & 287 & $1.96(0.73)$ & \\
\hline \multicolumn{4}{|l|}{ Lymph node metastasis } \\
\hline Negative & 358 & $1.72(0.82)$ & \\
\hline Positive & 6 & $2.42(0.77)$ & \\
\hline \multicolumn{4}{|l|}{ High grade PIN } \\
\hline Negative & 3 & $0.63(0.58)$ & \\
\hline Positive & 360 & $1.74(0.82)$ & \\
\hline
\end{tabular}

PIN, prostatic intraepithelial neoplasia.

${ }^{*} P$-value reported is from a $T$-test or Analysis of Variance (ANOVA).

mean tumor diameter was $1.73 \mathrm{~cm}$ (range, 0.20 $4.40 \mathrm{~cm})$. The mean tumor volume was $2.50 \mathrm{~cm}^{3}$ (range, $0.01-38.00 \mathrm{~cm}^{3}$ ). Gleason scores were $4(1$, $0.3 \%), 5(63,17.3 \%), 6(114,31.3 \%), 7$ (151, $41.5 \%)$, $8(9,2.5 \%), 9(26,7.1 \%)$. The mean Gleason score was 6.5. Tumors were multifocal in $85 \%$ of the cases and $23 \%$ of the cases had positive surgical margins.

Biochemical disease as determined by PSA recurrence was identified in 45 of the 364 patients $(12 \%)$ following radical prostatectomy. In patients with PSA failure, the median time to recurrence was 3 months and the mean time to recurrence was 7 months (range, 3-30 months). PSA recurrence was identified in $5 \%$ of patients with maximum tumor diameter $\leq 1.7 \mathrm{~cm}$, compared to $20 \%$ of patients with maximum tumor diameter $>1.7 \mathrm{~cm}$. Recurrence occurred in $4 \%$ of tumors with Gleason score $4-6,13 \%$ of tumors with Gleason score 7, and $51 \%$ of tumors with Gleason score 8-9. Gleason score was 7 or greater in $84 \%$ of tumors with recurrence.

Maximum tumor diameter was associated with preoperative PSA $(r=0.22, P<0.0001)$, prostate weight ( $r=-0.12, P=0.03)$, tumor volume $(r=0.87$, $P<0.0001)$, Gleason score $(r=0.29, P<0.0001)$, and pathologic stage $(P<0.0001)$. For analysis, pathological stage was defined as organ confined (pT2a and pT2b combined), extraprostatic extension (pT3a) and seminal vesicle invasion (pT3b). Both extraprostatic extension and seminal vesicle invasion were associated with significantly higher maximum tumor diameter compared to those with organconfined disease. Maximum tumor diameter was also associated with positive surgical margins and perineural invasion (Table 2). In Cox multiple regression, maximum tumor diameter was tested for predictive value adjusting for pathological stage, extraprostatic extension, seminal vesicle invasion, surgical margin status, Gleason score, and maximum tumor diameter. Pathological stage with $P>0.01$ between all categories was eliminated from the model. The reduced model with predictor maximum tumor diameter adjusted for sugical margins and Gleason score is presented in Table $3(P=0.01,0.084$ and $<0.0001$, respectively). As a measure of fit for Cox regression to compare the models, Akaike's information criterion (AIC) was used with better fit 
Table 3 Reduced Cox multiple regression predicting time to PSA recurrence using maximum tumor diameter and other covariates*

\begin{tabular}{lccc}
\hline Variable & Adjusted hazard ratio & 95\% confidence interval & P-value \\
\hline Surgical margin status & 1.78 & $(0.93,3.43)$ & 0.084 \\
Gleason score & 1.90 & $(1.45,2.48)$ & $<.0001$ \\
Maximum tumor diameter $(\mathrm{cm})$ & 1.70 & $(1.13,2.56)$ & 0.011 \\
\hline
\end{tabular}

*Terms remaining in the reduced model have $P$-values $<0.10$ (see Results section).

corresponding to smaller AIC. ${ }^{15}$ The full model $\mathrm{AIC}=310$ was greater than the reduced model $\mathrm{AIC}=307$ indicating better fit of the reduced model. Maximum tumor diameter was found to be a significant predictor of time to recurrence adjusting for covariates Gleason score and surgical margins. These results suggest that each $\mathrm{cm}$ increase in maximum tumor diameter is associated with a $70 \%$ increase in risk of recurrence (hazard ratio $=$ 1.70; 95\% confidence interval 1.13-2.56).

\section{Discussion}

In this series of 364 totally embedded, serially sectioned, whole-mount radical prostatectomy specimens, maximum tumor diameter was a significant predictor of biochemical recurrence. Maximum tumor diameter was also associated with preoperative PSA, prostate weight, tumor volume, Gleason score, pathologic stage, presence of extraprostatic extension, seminal vesicle invasion, surgical margins, and perineural invasion.

Several studies have assessed prostate cancer progression in association with morphologic and clinical variables. Stamey et $a l^{16}$ noted that tumor volume (as determined by computer planimetry), percent Gleason grade 4-5, positive lymph nodes, and intraprostatic vascular invasion were associated with progression as defined by increasing PSA level. ${ }^{16}$ Tumor progression occurred in $14 \%$ of men with tumor volumes in the range of $0.5-2.0 \mathrm{~cm}^{3}$, compared to $97 \%$ of men with tumor volumes greater than $12.0 \mathrm{~cm}^{3}$.

In a series of 57 patients undergoing radical prostatectomy, Renshaw et $a l^{17}$ found that maximum tumor diameter predicted PSA failure, but had marginal statistical significance as a risk factor for PSA failure (risk ratio $=1.12$ ). Even so, no patient with a tumor less than $1 \mathrm{~cm}$ in maximum diameter experienced recurrence, while all patients with tumors greater than $2 \mathrm{~cm}$ experienced recurrence. ${ }^{17}$ In a later study with 434 patients, of whom $27 \%$ experienced PSA failure, maximum tumor diameter was found to be a significant predictor of PSA failure, as well as preoperative serum PSA level and Gleason score. ${ }^{18}$ In this larger study, $15 \%$ of men with a maximum tumor diameter less than $1 \mathrm{~cm}$ experienced failure, compared to $73 \%$ of men with maximum tumor diameter greater than $2 \mathrm{~cm}$. A limitation of both studies was the lack of uniform processing of the radical prostatectomy specimens, allowing for variation in assessment of prognostic factors.

In the current study, which has doubled the population size, we confirm previous findings of correlation between maximum tumor diameter and tumor volume, as well as other predictors of clinical outcome including surgical margin status, preoperative PSA level, Gleason score, and pathologic stage. ${ }^{6}$ In contrast to previous studies investigating maximum tumor diameter, our study evaluated totally embedded, serially sectioned, whole-mount prostatectomies that were uniformly processed. Wholemount processing eliminated the need for multiple slides per cross section, reducing concern that areas of contiguous tumor were separated and interpreted as distinct foci on routinely processed slides. While maintaining cross-sectional orientation is easier with whole-mount processing, maximum tumor diameter may also be accurately measured by routine sectioning. ${ }^{5}$

Biochemical recurrence may occur in up to $35 \%$ of men within 10 years following radical prostatectomy. ${ }^{19}$ Additional studies have reported a $15 \%$ biochemical recurrence rate after a median followup of 5 years, ${ }^{20}$ a $13 \%$ recurrence rate at 2 years, ${ }^{21}$ or $22 \%$ at 5 years. ${ }^{22}$ Of those men with biochemical recurrence, $34 \%$ will ultimately develop metastatic disease. $^{20}$ We observed a biochemical recurrence rate of $12 \%$ at 2 years in the current study population of 364 patients, which is comparable to these previous reports despite our relatively short interval of follow-up after radical prostatectomy. The majority of cases with recurrence had high Gleason scores ( $84 \%$ had a Gleason score $\geq 7$ ). Extended follow-up is needed to confirm these findings and more fully assess the extent of PSA recurrence, especially with tumors of lower Gleason score. Nonetheless, identification of patients with early treatment failure is important for patient management.

Maximum tumor diameter is a significant predictor of biochemical recurrence, and correlates with preoperative PSA, prostate weight, tumor volume, Gleason score, and pathologic stage, and predicts biochemical recurrence independent of these parameters. Inclusion of maximum tumor diameter in surgical pathology reports for radical prostatectomies should be considered. 


\section{References}

1 Renshaw AA. Correlation of gross morphologic features with histologic features in radical prostatectomy specimens. Am J Clin Pathol 1998;110:38-42.

2 Cheng L, Song SY, Pretlow TG, et al. Evidence of independent origin of multiple tumors from patients with prostate cancer. J Natl Cancer Inst 1998;90:233-237.

3 Greene DR, Egawa S, Neerhut G, et al. The distribution of residual cancer in radical prostatectomy specimens in stage A prostate cancer. J Urol 1991;146:1069-1076.

4 Arora R, Koch MO, Eble JN, et al. Heterogeneity of Gleason grade in multifocal adenocarcinoma of the prostate. Cancer 2004;100:2362-2366.

5 Renshaw AA, Chang H, D'Amico AV. Estimation of tumor volume in radical prostatectomy specimens in routine clinical practice. Am J Clin Pathol 1997;107: 704-708.

6 Eichelberger LE, Koch MO, Daggy JK, et al. Predicting tumor volume in radical prostatectomy specimens from patients with prostate cancer. Am J Clin Pathol 2003;120:386-391.

7 Babaian RJ, Troncoso P, Bhadkamkar VA, et al. Analysis of clinicopathologic factors predicting outcome after radical prostatectomy. Cancer 2001;91: 1414-1422.

8 van den Ouden D, Bentvelsen FM, Boeve ER, et al. Positive margins after radical prostatectomy: correlation with lacal recurrence and distant progression. Br J Urol 1993;72:489-494.

9 Koch MO, Foster RS, Bell B, et al. Characterization and predictors of prostate specific antigen progression rates after radical retropubic prostatectomy. J Urol 2000;164: 749-753.

10 Eichelberger LE, Cheng L. Does pT2b prostate carcinoma exist? Critical appraisal of the 2002 TNM classification of prostate carcinoma. Cancer 2004;100: 2573-2576.

11 Fleming ID, Cooper JS, Henson DE, et al. American Joint Committee on Cancer Staging Manual. Lippincott Raven: Philadelphia, 1997, pp 219-222.
12 Gleason DF, Mellingerr GT. Prediction of prognosis for prostatic adenocarcinoma by combined histologic grading and clinical stage. J Urol 1974;111:58-64.

13 Bostwick DG. Neoplasms of the prostate. In: Bostwick DG, Eble JN (eds). Urologic Surgical Pathology. Mosby: St Louis, 1997, pp 343-422.

14 Cheng L, Slezak J, Bergstralh EJ, et al. Preoperative prediction of surgical margin status in prostate cancer patients treated by radical prostatectomy. J Clin Oncol 2000;18:2862-2868.

15 Sakamoto Y, Ishiguro M, Kitagawa G. Akaike Information Criterion Statistics. D. Reidel Publishing Company: The Netherlands, 1986.

16 Stamey T, McNeal J, Yemoto C, et al. Biological determinants of cancer progression in men with prostate cancer. JAMA 1999;281:1395-1400.

17 Renshaw AA, Richie JO, Loughlin KR, et al. The greatest dimension of prostate carcinoma is a simple, inexpensive predictor of prostate specific antigen failure in radical prostatectomy specimens. Cancer 1998;83:748-752.

18 Renshaw AA, Richie JP, Loughlin KR, et al. Maximum diameter of prostatic carcinoma is a simple, inexpensive, and independent predictor of prostate-specific antigen failure in radical prostatectomy specimens. Am J Clin Pathol 1999;111:641-644.

19 Han M, Partin AW, Pound CR, et al. Long-term biochemical disease-free and cancer-specific survival following anatomic radical retropubic prostatectomy. Urol Clin N Am 2001;28:555-565.

20 Pound CR, Partin AW, Eisenberger MA, et al. Natural history of progression after PSA elevation following radical prostatectomy. JAMA 1999;281:1591-1597.

21 Obek C, Sadek S, Lai S, et al. Positive surgical margins with radical retropubic prostatectomy: anatomic sitespecific pathologic analysis and impact on prognosis. Urology 1999;54:682-688.

22 Catalona WJ, Smith DS. Cancer recurrence and survival rates after anatomic radical retropubic prostatectomy for prostate cancer: intermediate-term results. J Urol 1998;160:2428-2434. 